\title{
Rare mutations provide unique insight into oncogenic potential of STAT transcription factors
}

\author{
Lisa N. Heppler and David A. Frank \\ Department of Medical Oncology, Dana-Farber Cancer Institute, Department of Medicine, Brigham and Women's Hospital and Harvard Medical School, Boston, Massachusetts, USA.
}

\begin{abstract}
The inappropriate activation of transcription factors, including STATs, is known to promote tumor initiation and progression. The most common mechanisms of misregulation lead to constitutive activation of WT STATs. However, the recent discovery of rare STAT mutations in hematopoietic malignancies suggests that STAT mutants may be oncogenic. In this issue of the $J C l$, Pham et al. use a transgenic mouse model to demonstrate that STAT5B ${ }^{\mathrm{N} 642 \mathrm{H}}$ is sufficient for the development of T cell neoplasia. This study, along with other studies of constitutively active STAT mutants, provides insight into the pathogenesis and treatment of STAT5-driven cancer.
\end{abstract}

\section{Oncogenic transcription factors}

Many human diseases are characterized by aberrant gene expression patterns that alter cellular function. Such abnormal expression is commonly caused by the misregulation of transcription factors, transcriptional cofactors, and chromatin regulators (1). Within the context of cancer, misregulation often manifests as inappropriate activation of unmutated or WT transcription factors that control the expression of genes promoting proliferation and survival (2). These mechanisms of activation often include overexpression or hyperactivation of the WT protein. Although less common, misregulation can also manifest as gainof-function point mutations in the gene encoding the transcription factor $(3,4)$. Regardless of the mechanism, these events can lead to the inappropriate activation of transcription factors that promote tumor initiation and progression, thus transforming normal cellular proteins into so-called "oncogenic" transcription factors.

\section{STATs in cancer}

One prototypical family of oncogenic transcription factors, the STAT family, consists of seven members, STAT1 through STAT6, including the closely related STAT5A and STAT5B. In the absence of an upstream signal, such as cytokine or growth factor stimulation, STATs reside in the cytoplasm as latent, antiparallel dimers. Following stimulation, STATs are tyrosine phosphorylated by upstream kinases, including JAKs, and undergo a conformational change to form active, parallel dimers that translocate to the nucleus and regulate the expression of genes involved in proliferation, differentiation, and survival (Figure 1). Active STAT dimers are then dephosphorylated and shuttled out of the nucleus to complete the activation-inactivation cycle (5).

Under physiological conditions, STAT signaling is transient and tightly regulated by endogenous inhibitors, such as suppressor of cytokine signaling proteins, protein inhibitor of activated STAT proteins, and protein tyrosine phosphatases. However, because of the proproliferation and prosurvival activity of STAT target genes, STAT misregulation can promote tumor initiation and progression. The most common mechanism of STAT misregulation is persistent STAT activation due to chronically elevated cytokine levels, loss of endogenous inhibitors, or hyperactivation of upstream signal-
Related Article: p. 387

Conflict of interest: D.A. Frank receives research support from Gilead Sciences and is the inventor on US Patent $8,445,517$ "Stat modulators." The spouse of D.A. Frank is employed by and owns shares in Biogen. Reference information: / Clin Invest. 2018;128(1):113-115. https://doi.org/10.1172/JCI98619. ing pathway components, such as receptors and tyrosine kinases $(6,7)$. Another mechanism of misregulation that has become more apparent in recent years is gain-of-function point mutations within STAT-encoding genes (Figure 1). Although rare within the patient population, these STAT mutations provide a unique opportunity to directly test whether STAT transcription factors are necessary or sufficient for oncogenesis.

\section{Gain-of-function STAT mutations}

Initial efforts to generate and characterize gain-of-function STAT mutations in the laboratory used both random and structure-driven mutagenesis. Onishi et al. (8) used PCR-driven mutagenesis to introduce random mutations into the STAT5A sequence and then used retroviral delivery to introduce these mutated STAT5A sequences into $\mathrm{Ba} / \mathrm{F} 3$ cells, the murine pro-B cells that normally depend on IL-3 for proliferation and survival, but become IL-3 independent following transformation. Thus, using IL-3-independent growth as a proxy for STAT5-dependent oncogenesis, Onishi and colleagues identified a constitutively active STAT5 mutant, STAT5A1*6, with two amino acid substitutions, one in the DNA-binding domain (H299R) and another in the transactivation domain (S711F). Moreover, they demonstrated that this STAT5 mutant was constitutively tyrosine phosphorylated, localized in the nucleus, and transcriptionally active, even in the absence of cytokine.

A few years later, the same group identified another constitutively active STAT5 mutant known as STAT5A ${ }^{\mathrm{N} 642 \mathrm{H}}$ (9). Unlike STAT5A1 $^{* 6}$, STAT5A ${ }^{\mathrm{N} 642 \mathrm{H}}$ harbors an amino acid substitution in the $\mathrm{SH} 2$ domain that restores a conserved histidine known to be required for SH2-mediated phosphotyrosine binding in other proteins. Accordingly, Ariyoshi and associates demonstrated that mutating Y694, which is required for STAT5 phosphorylation and activation, negates the gain-of-function activity of STAT5 $\mathrm{A}^{\mathrm{N} 642 \mathrm{H}}$, 


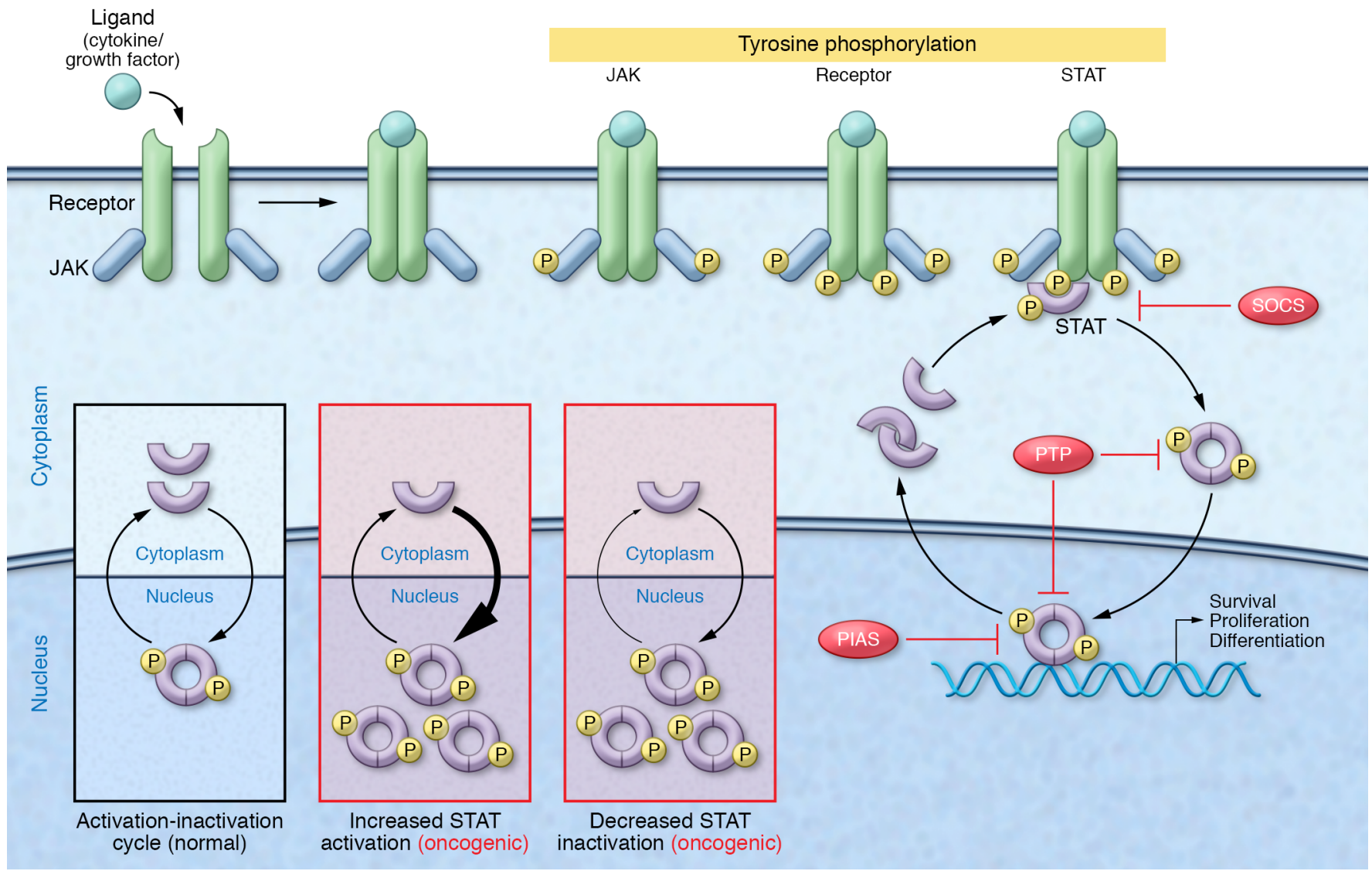

Figure 1. STAT-mediated oncogenesis. Under physiologic conditions, STAT signaling is stimulus dependent and tightly regulated by endogenous inhibitors, including SOCS proteins, protein inhibitor of activated STAT (PIAS) proteins, and protein tyrosine phosphatases (PTPs). Cytokine or growth factor stimulation triggers receptor oligomerization and sequential tyrosine phosphorylation of receptor-associated JAKs, intracellular receptor domains, and newly recruited STAT proteins. Phosphorylated STAT dimers then translocate to the nucleus, where they bind DNA and control the expression of genes regulating proliferation, survival, and differentiation. Following dephosphorylation, STATs are shuttled back out of the nucleus, completing the activation-inactivation cycle. Cancer-associated events can lead to constitutive STAT activity and STAT-dependent oncogenesis. This can occur through increased STAT activation due to elevated cytokine levels, loss of endogenous inhibitors, or hyperactivation of upstream signaling machinery, or via decreased STAT inactivation, such as that occurring through loss of endogenous inhibitors and dephosphorylation-resistant STAT mutants. The STAT5 mutant STAT5B ${ }^{\mathrm{N} 642 \mathrm{H}}$ analyzed by Pham et al. (18) appears to drive the malignant transformation of hematopoietic cells through this latter mechanism.

clearly indicating that tyrosine phosphorylation and dimerization are necessary for the constitutive activity of STAT5A $\mathrm{A}^{\mathrm{N} 642 \mathrm{H}}$. Interestingly, they also found that the same mutation renders STAT5B constitutively active. Together, the studies on STAT5A1*6 and STAT5 $\mathrm{A}^{\mathrm{N} 642 \mathrm{H}}$ demonstrate that constitutively active STAT5 mutants are oncogenic in vitro and are activated through processes that mirror physiological STAT activation.

Around the same time, Bromberg et al. (10) used a structure-based approach to generate and characterize constitutively active STAT3 molecules. In brief, they used crystal structures of DNA-bound STAT dimers to identify two residues within the $\mathrm{SH} 2$ domain that could be exchanged for cysteine to facilitate the formation of sulfhydryl bonds between STAT3 monomers. They hypothesized that such modifica- tions, specifically at A661 and N663, would render the STAT3 molecules constitutively active by promoting STAT3 dimerization, even in the absence of STAT3 tyrosine phosphorylation. Using site-directed mutagenesis, they engineered the disulfide-containing constitutively active STAT3 mutant known as STAT3C. They demonstrated that STAT3C is constitutively active and able to cause neoplastic transformation of fibroblasts, which could then form tumors in nude mice. Moreover, they showed that the DNA-binding activity of STAT3C was inhibited by reducing agents, indicating that the hypothesized disulfide bonds are indeed important for STAT3C activity. However, they did not specifically test whether the presence of cysteine residues was sufficient for constitutive STAT3 activity in the absence of tyrosine phosphorylation.
To address this critical question, Liddle et al. (11) developed STAT3C mutants that were unable to be tyrosine phosphorylated. Interestingly, they found that mutating the critical tyrosine residue Y705 abrogated the transcriptional activity of STAT3C, demonstrating that disulfide-bond formation between STAT3 monomers is not sufficient for STAT3 activation. Moreover, Liddle and colleagues showed that STAT3C tyrosine phosphorylation was significantly prolonged following cytokine stimulation as compared with WT STAT3, suggesting that the constitutive activity of STAT3C is a result of increased dimer stability and reduced dephosphorylation rather than phosphorylation-independent dimerization. Overall, these studies with STAT3C recapitulate the STAT5A1* 6 and STAT5A $^{\mathrm{N} 642 \mathrm{H}}$ findings, strongly suggesting 
that constitutively active STAT mutants are not uniquely activated, but rather uniquely resistant to inactivation (Figure 1).

The identification and characterization of artificial STAT mutants raised the possibility that constitutively active STAT mutants may drive the pathogenesis of human cancer in patients. Rajala et al. (12) first discovered somatic STAT5 mutations in a small fraction of patients (4 of 211) with large granular lymphocytic (LGL) leukemia, a chronic leukemia characterized by the expansion of cytotoxic T cells or NK cells. They identified two mutations, STAT5B ${ }^{\mathrm{Y} 665 \mathrm{~F}}$ and STAT5B ${ }^{\mathrm{N} 642 \mathrm{H}}$, both of which were found in the $\mathrm{SH} 2$ domain and led to an increase in STAT5B phosphorylation and transcriptional activity. Additionally, the presence of STAT5B ${ }^{\mathrm{N} 642 \mathrm{H}}$ correlated with an abnormally aggressive and fatal disease compared with typical LGL leukemia.

Rare activating STAT5B mutations have since been found in other hematologic malignancies, including $\mathrm{T}$ cell acute lymphoblastic leukemia (T-ALL), NK/T cell lymphomas of the nasal type (NKTCLs), $\gamma \delta$ peripheral $\mathrm{T}$ cell lymphomas ( $\gamma \delta$-PTCLs), $\mathrm{T}$ cell prolymphocytic leukemia (T-PLL), and $\gamma \delta$ hepatosplenic $\mathrm{T}$ cell lymphomas $(13-17)$. In this issue of the JCI, Pham et al. (18) use a transgenic mouse model to directly test whether STAT5B ${ }^{\mathrm{N} 642 \mathrm{H}}$, the most frequent STAT5B mutation, is capable of driving the development and progression of human leukemia. First, they observed that STAT5B $^{\mathrm{N} 642 \mathrm{H}}$ enhances STAT5 tyrosine phosphorylation in vitro and renders $\mathrm{Ba} / \mathrm{F} 3$ and 32D cells cytokine independent, recapitulating many of the initial findings concerning the artificial STAT mutants $(8,9,11)$.

Next, Pham et al. (18) used the Vav1 oncogene promoter system first described by Ogilvy et al. (19) to preferentially express either WT STAT5B or mutant STAT5B ${ }^{\mathrm{N} 642 \mathrm{H}}$ in the hematopoietic compartment. They found that transgenic mice expressing human STAT5B ${ }^{\mathrm{N} 642 \mathrm{H}}$ developed aggressive T cell leukemia/lymphoma (TLL) characterized by persistent tyrosine phosphorylation, increased numbers of myeloid progenitor cells, splenomegaly, and increased WBC counts, including expansion of $\mathrm{CD} 8^{+} \mathrm{T}$ cells. Moreover, they showed that transplantation of $\mathrm{CD}^{+} \mathrm{T}$ cells from STAT5B ${ }^{\mathrm{N} 642 \mathrm{H}}$-transgenic mice into nonirradiated recipients phenotypically recapitulated the primary disease. In contrast, WT STAT5B-transgenic mice showed no signs of disease.

Given the persistent STAT5 tyrosine phosphorylation seen both in vitro and in the STAT5B ${ }^{\mathrm{N} 642 \mathrm{H}}$-transgenic mouse model, Pham and associates (18) tested whether FDA-approved JAK inhibitors, such as ruxolitinib and tofacitinib, could suppress STAT5B $\mathrm{B}^{\mathrm{N} 642 \mathrm{H}}$-driven disease. In support of previous findings from Liddle et al. (11), treating STAT5B ${ }^{\mathrm{N} 642 \mathrm{H}}$-transgenic mice with ruxolitinib decreased STAT5 tyrosine phosphorylation and markedly reduced disease burden, as measured by lymph node and spleen size, WBC counts, and T cell infiltration in lung, skin, and bone marrow. Together, these findings not only demonstrate that STAT5 $\mathrm{B}^{\mathrm{N} 642 \mathrm{H}}$ is a driver mutation in TLL, but also highlight the utility of the Vav1 preclinical model for testing the efficacy of treatment regimens for patients carrying STAT5B mutations.

\section{Therapeutic implications}

Although this study by Pham et al. (18) focuses on STAT5B ${ }^{\mathrm{N} 642 \mathrm{H}}$-driven $\mathrm{T}$ cell neoplasia, the findings are likely to have broader therapeutic implications. Constitutively active STAT5 mutants appear to be activated through processes that recapitulate physiologic STAT activation and are oncogenic as a result of persistent tyrosine phosphorylation and transcriptional activation of STAT5 target genes. Thus, therapeutic regimens that demonstrate efficacy in WT STAT5driven disease will likely demonstrate efficacy in mutant STAT5-driven disease, and vice versa. These observations have considerable implications for therapeutic development and suggest that STAT5-specific inhibitors such as pimozide that alter tyrosine phosphorylation may show activity in mutant STAT5B-driven cancer, including STAT5B $^{\mathrm{N} 642 \mathrm{H}}$-driven $\mathrm{T}$ cell neoplasia $(20,21)$.

Address correspondence to: David A. Frank, Department of Medical Oncology, Dana-Farber Cancer Institute, 450 Brookline Avenue, Boston, Massachusetts 02215, USA. Phone: 617.632.4714; Email: david_frank@dfci.harvard.edu.

1. Lee TI, Young RA. Transcriptional regulation and its misregulation in disease. Cell. 2013;152(6):1237-1251.

2. Yeh JE, Toniolo PA, Frank DA. Targeting transcription factors: promising new strat- egies for cancer therapy. Curr Opin Oncol. 2013;25(6):652-658.

3. Darnell JE Jr. Transcription factors as targets for cancer therapy. Nat Rev Cancer. 2002;2(10):740-749.

4. Bhagwat AS, Vakoc CR. Targeting transcription factors in cancer. Trends Cancer. 2015;1(1):53-65.

5. Levy DE, Darnell JE Jr. Stats: transcriptional control and biological impact. Nat Rev Mol Cell Biol. 2002;3(9):651-662.

6. Vainchenker W, Constantinescu SN. JAK/STAT signaling in hematological malignancies. Oncogene. 2013;32(21):2601-2613.

7. Quintás-Cardama A, Verstovsek S. Molecular pathways: Jak/STAT pathway: mutations, inhibitors, and resistance. Clin Cancer Res. 2013;19(8):1933-1940.

8. Onishi M, et al. Identification and characterization of a constitutively active STAT5 mutant that promotes cell proliferation. Mol Cell Biol. 1998;18(7):3871-3879.

9. Ariyoshi K, et al. Constitutive activation of STAT 5 by a point mutation in the $\mathrm{SH} 2$ domain. J Biol Chem. 2000;275(32):24407-24413.

10. Bromberg JF, et al. Stat3 as an oncogene. Cell. 1999;98(3):295-303.

11. Liddle FJ, Alvarez JV, Poli V, Frank DA. Tyrosine phosphorylation is required for functional activation of disulfide-containing constitutively active STAT mutants. Biochemistry. 2006;45(17):5599-5605.

12. Rajala HL, et al. Discovery of somatic STAT5b mutations in large granular lymphocytic leukemia. Blood. 2013;121(22):4541-4550.

13. Kontro M, et al. Novel activating STAT5B mutations as putative drivers of T-cell acute lymphoblastic leukemia. Leukemia. 2014;28(8):1738-1742.

14. Küçük C, et al. Activating mutations of STAT5B and STAT3 in lymphomas derived from $\gamma \delta$-T or NK cells. Nat Commun. 2015;6:6025.

15. Kiel MJ, et al. Integrated genomic sequencing reveals mutational landscape of T-cell prolymphocytic leukemia. Blood. 2014;124(9):1460-1472.

16. López C, et al. Genes encoding members of the JAK-STAT pathway or epigenetic regulators are recurrently mutated in T-cell prolymphocytic leukaemia. Br J Haematol. 2016;173(2):265-273.

17. Nicolae A, et al. Frequent STAT5B mutations in $\gamma \delta$ hepatosplenic T-cell lymphomas. Leukemia. 2014;28(11):2244-2248.

18. Pham HTT, et al. STAT5 $\mathrm{B}^{\mathrm{N} 642 \mathrm{H}}$ is a driver mutation for $\mathrm{T}$ cell neoplasia. J Clin Invest. 2018;128(1):387-401.

19. Ogilvy S, Metcalf D, Gibson L, Bath ML, Harris AW, Adams JM. Promoter elements of vav drive transgene expression in vivo throughout the hematopoietic compartment. Blood. 1999;94(6):1855-1863.

20. Nelson EA, et al. The STAT5 inhibitor pimozide decreases survival of chronic myelogenous leukemia cells resistant to kinase inhibitors. Blood. 2011;117(12):3421-3429.

21. Nelson EA, et al. The STAT5 inhibitor pimozide displays efficacy in models of acute myelogenous leukemia driven by FLT3 mutations. Genes Cancer. 2012;3(7-8):503-511. 